\title{
Orbital variability of the PSR J2051-0827 binary system
}

\author{
O. Doroshenko ${ }^{1}$, O. Löhmer ${ }^{1}$, M. Kramer ${ }^{2}$, A. Jessner ${ }^{1}$, R. Wielebinski ${ }^{1}$, A. G. Lyne ${ }^{2}$, and Ch. Lange ${ }^{1}$ \\ 1 Max-Planck-Institut für Radioastronomie, Auf dem Hügel 69, 53121 Bonn, Germany \\ ${ }^{2}$ University of Manchester, Jodrell Bank Observatory, Macclesfield, Cheshire SK11 9DL, UK
}

Received 21 May 2001 / Accepted 26 September 2001

\begin{abstract}
We have carried out high-precision timing measurements of the binary millisecond pulsar PSR J2051-0827 with the Effelsberg 100-m radio telescope of the Max-Planck-Institut für Radioastronomie and with the Lovell $76-\mathrm{m}$ radio telescope at Jodrell Bank. The 6.5-yrs radio timing measurements have revealed a significant secular variation of the projected semi-major axis of the pulsar at a rate of $\dot{x} \equiv \mathrm{d}\left(a_{1} \sin i\right) / \mathrm{d} t=$ $(-0.23 \pm 0.03) \times 10^{-12}$, which is probably caused by the Newtonian spin-orbit coupling in this binary system leading to a precession of the orbital plane. The required misalignment of the spin and orbital angular momenta of the companion are evidence for an asymmetric supernova explosion. We have also confirmed that the orbital period is currently decreasing at a rate of $\dot{P}_{\mathrm{b}}=(-15.5 \pm 0.8) \times 10^{-12} \mathrm{~s} \mathrm{~s}^{-1}$ and have measured second and third orbital period derivatives $\mathrm{d}^{2} P_{\mathrm{b}} / \mathrm{d} t^{2}=(+2.1 \pm 0.3) \times 10^{-20} \mathrm{~s}^{-1}$ and $\mathrm{d}^{3} P_{\mathrm{b}} / \mathrm{d} t^{3}=(3.6 \pm 0.6) \times 10^{-28} \mathrm{~s}^{-2}$, which indicate a quasi-cyclic orbital period variation similar to those found in another eclipsing pulsar system, PSR B1957+20. The observed variation of the orbital parameters constrains the maximal value of the companion radius to $R_{\mathrm{c} \max } \sim 0.06 R_{\odot}$ and implies that the companion is underfilling its Roche lobe by $50 \%$. The derived variation in the quadrupole moment of the companion is probably caused by tidal dissipation similar to the mechanism proposed for PSR B1957+20. We conclude that the companion is at least partially non-degenerate, convective and magnetically active.
\end{abstract}

Key words. relativity - pulsars: general - stars: neutron - stars: individual (PSR J2051-0827)

\section{Introduction}

The eclipsing binary millisecond pulsar PSR J2051-0827 was discovered with the Parkes $64-\mathrm{m}$ radio telescope in Australia in a $0.4 \mathrm{GHz}$ survey of the southern sky (Stappers et al. 1996a). This system has one of the shortest known orbital periods, $P_{\mathrm{b}} \simeq 2.4 \mathrm{hrs}$, and is moving in an almost circular, compact orbit, with a separation between the pulsar and its companion of only $1.03 R_{\odot}$. Observations of the pulsar with the 64-m Parkes and the 76-m Lovell radio telescope at Jodrell Bank at frequencies between $0.4 \mathrm{GHz}$ and $2 \mathrm{GHz}$ showed that at lower frequencies near $0.6 \mathrm{GHz}$ the pulsar is eclipsed by the atmosphere of the companion during approximately $10 \%$ of the orbital period, whereas at higher frequencies near $1.4 \mathrm{GHz}$ there are almost no visible eclipses in this system (Stappers et al. 1996a). High precision timing observations of PSR J2051-0827 revealed that the orbital period of the system is decreasing at a rate of $\dot{P}_{\mathrm{b}} \sim-10^{-11} \mathrm{~s} \mathrm{~s}^{-1}$ indicating a decay time of the system of only $25 \mathrm{Myr}$ (Stappers et al. 1998). Early optical observations of the field of

Send offprint requests to: O. Löhmer,

e-mail: loehmer@mpifr-bonn.mpg.de
PSR J2051-0827 revealed that the amplitude of the companion's light curve is about $1.2 \mathrm{mag}$ and the companion is probably rotating synchronously around the pulsar so that one side is being heated by the impinging pulsar flux (Stappers et al. 1996b). The fit of photometry data to a model of a gravitationally distorted, low-mass secondary star that is irradiated by the impinging pulsar wind, has shown that the inclination of the system is greater than $30^{\circ}$ and the maximum companion mass is $0.055 M_{\odot}$ (Stappers et al. 1999). Recent observations of the pulsar's companion using the Hubble Space Telescope (HST) Wide Field Planetary Camera have allowed to detect its "dark" side by Stappers et al. (2001b). Surprisingly, they detected a slight asymmetry in the companion's light curve indicating that a simple synchronously rotating companion is no longer a complete model. Fitting the same model as Stappers et al. (1999) indicated that more than $30 \%$ of the pulsar spin-down energy is converted into optical emission and that the system is moderately inclined, i.e. $i \sim 40^{\circ}$.

Long-term timing observations of eclipsing (and hence often interacting) binary pulsars can reveal additional variations in orbital elements, which are important for understanding the evolution models of these systems and 
of pulsars in general. The orbital period derivative may change with time in a quasi-periodic way similar to PSR B1957+20 (Arzoumanian et al. 1994) or may remain unchanged over longer time intervals like for PSR B174424A (Nice et al. 2000).

We have carried out high precision timing observations of the binary system PSR J2051-0857 during the last 6.5 years. In addition to improving previously determined timing parameters, we detect, for the first time, time derivatives of the orbital period of second and third order and the time derivative of the semi-major axis. We use these discovered orbital variations to constrain the size of the pulsar companion. We discuss possible additional relativistic and non-relativistic effects that could be the cause for the apparent variations of the orbit.

\section{Observations}

PSR J2051-0827 was observed with both the 100$m$ radiotelescope of the Max-Planck-Institut für Radioastronomie in Effelsberg and the 76-m Lovell radiotelescope at Jodrell Bank. Observations have been carried out since 1996 using the Effelsberg 100-m radio telescope soon after the discovery of the pulsar with the 64-m Parkes radio telescope in Australia. Timing data have been acquired approximately once per month, with a few larger gaps due to unavailability of telescope time. We have collected about 350 individual times-of-arrival (TOAs) at center frequencies of $0.86,1.4,1.7$ and $2.7 \mathrm{GHz}$. Most of the observations were performed at frequencies near $1.4 \mathrm{GHz}$.

During a period in August-October 1996, the data were obtained using the Effelsberg Pulsar Observation System (EPOS). Here two channels of circular polarization are processed in a polarimeter and a $4 \times 60 \times 666 \mathrm{KHz}$ filter bank combined with an incoherent hardware dedisperser (Kramer et al. 1997, 1998). Only the two orthogonal total power signals of $40 \mathrm{MHz}$ bandwidth were used for later analysis. The input signal from the radio telescope is synchronously accumulated into 1024 pulse bins with the apparent pulsar spin period. The time stamp of each integration is synchronised to time signals from a hydrogen maser clock calibrated with the signals from the Global Positioning System (GPS). The dedispersed pulsar profiles were integrated for $15 \mathrm{~s}$ and later transformed to $8 \mathrm{~min}$ - integrations for further template matching in the time domain, using the cross-correlation of the integrated profile with a high signal-to-noise template.

Since October 1996, the data were collected using the Effelsberg - Berkeley Pulsar Processor (EBPP), a coherent dispersion removal processor, installed at Effelsberg (Backer et al. 1997). The EBPP provides 32 channels for both polarizations with a total bandwidth of up to $112 \mathrm{MHz}$ depending on the observing frequency and dispersion measure. For PSR J2051-0827 bandwidths of $28 \mathrm{MHz}, 56 \mathrm{MHz}$ and $112 \mathrm{MHz}$ were available at $0.9 \mathrm{GHz}$, 1.4 GHz and $2.7 \mathrm{GHz}$ respectively. The outputs of each channel are fed into dedisperser boards for coherent
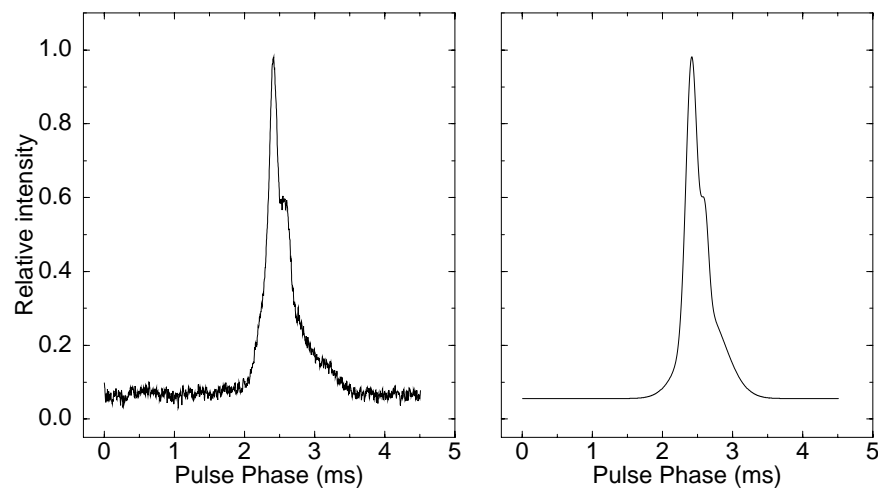

Fig. 1. Integrated profile of PSR J2051-0827, obtained with the EBPP at $1.4 \mathrm{GHz}$ (left) and the profile template, constructed from fitting of composition of three Gaussians to the integrated profile (right), which is used for template matching.

on-line dedispersion. All 64 output signals are integrated synchronously with the apparent pulsar period. The integrated profiles ( $5 \mathrm{~min}$ integration time) were matched in the frequency domain (Taylor 1991) with a synthetic template. The template itself consists of three Gaussian components (Fig. 1 right) that were fitted to a high signalto-noise pulsar profile (Fig. 1 left) using the method developed by Kramer et al. (1994, 1998). The time stamp of each integrated profile was provided by a hydrogen maser clock calibrated with signals from the GPS to the Universal Coordinated Time UTC(NIST).

In order to get a better timing solution for the orbital parameters of the system, the sets of TOAs in several observing sessions were acquired during one complete pulsar revolution around its orbit, equal to $\sim 2.4 \mathrm{hrs}$. Typical TOA uncertainties in observations are $\sim 8 \mu$ s at 1.4 and $1.7 \mathrm{GHz}, \sim 10 \mu$ s at $2.7 \mathrm{GHz}$ and $\sim 25 \mu$ s at $0.9 \mathrm{GHz}$.

The Jodrell Bank data were collected since August 1994 using cryogenic receivers at 0.3, 0.4, 0.6 and $1.4 \mathrm{GHz}$. The observations were made using both circular polarization directions utilising a $2 \times 64 \times 125 \mathrm{KHz}$ filter bank. The added output signals from both polarizations were filtered and digitised for further on-line hardware dedispersion and synchronous folding with the pulsar's apparent period. Integrated profiles with integration times of 3 min were matched by standard pulse template to obtain topocentric TOAs (Bell et al. 1997). Typical TOA uncertainties in these observations are $\sim 10 \mu$ s at 0.4 and $1.4 \mathrm{GHz}$ and $\sim 16 \mu$ s at 0.3 and $0.6 \mathrm{GHz}$.

\section{Data reduction and timing solution}

The obtained TOAs corrected to UTC(NIST) were fitted to a spin-down model of the pulsar rotation in the binary system using both the TIMAPR ${ }^{1}$ (Doroshenko \& Kopeikin 1995) and the TEMPO ${ }^{2}$ software package, which both use the DE200 ephemeris of the Jet Propulsion

\footnotetext{
1 http://www.mpifr-bonn.mpg.de/div/pulsar/former/ olegd/soft.html

2 http://pulsar.princeton.edu/tempo
} 
Table 1. Spin, astrometric, Keplerian and post-Keplerian parameters of binary pulsar system PSR J2051-0827.

\begin{tabular}{ll}
\hline Parameter & Value \\
\hline \multicolumn{1}{c}{ Astrometric, spin and medium } & parameters \\
Right ascension $\alpha(\mathrm{J} 2000)$ & $20^{\mathrm{h}} 51^{\mathrm{m}} 07^{\mathrm{s}} .5145(1)$ \\
Declination $\delta(\mathrm{J} 2000)$ & $-08^{\circ} 27^{\prime} 37^{\prime \prime} \cdot 795(5)$ \\
Proper motion in RA $\mu_{\alpha}\left(\mathrm{mas} \mathrm{yr}^{-1}\right)$ & $5.3(1.0)^{a}$ \\
Proper motion in Dec $\mu_{\delta}\left(\mathrm{mas} \mathrm{yr}^{-1}\right)$ & $0.3(3.0)^{a}$ \\
Spin period $P(\mathrm{~ms})$ & $4.5086417449716(2)$ \\
Period derivative $\dot{P}\left(\mathrm{~s} \mathrm{~s}^{-1}\right)$ & $1.2737(5) \times 10^{-20}$ \\
Spin frequency $\nu\left(\mathrm{s}^{-1}\right)$ & $221.796287344250(8)$ \\
Spin frequency derivative $\left.\dot{\nu}^{-2}\right)$ & $-6.266(2) \times 10^{-16}$ \\
Dispersion measure DM $\left(\mathrm{pc} \mathrm{cm}^{-3}\right)$ & $20.7449(4)$ \\
Derivative of DM, DM $\left(\mathrm{pc} \mathrm{cm}{ }^{-3} \mathrm{yr}^{-1}\right)$ & $0.0011(3)$ \\
Epoch (MJD) & 51000.0
\end{tabular}

Orbital period $P_{\mathrm{b}}(\mathrm{d})$

Parameters of binary orbit

Projected semi-major axis $x$ (lt-s)

Eccentricity $e$

Epoch of periastron $T_{0}$, (MJD)

Longitude of periastron, $\omega_{0}$

Orbital period derivative $\dot{P}_{\mathrm{b}}\left(\mathrm{s} \mathrm{s}^{-1}\right)$

Second derivative of $P_{\mathrm{b}}, \ddot{P}_{\mathrm{b}}\left(\mathrm{s}^{-1}\right)$

Third derivative of $P_{\mathrm{b}}, \mathrm{d}^{3} P_{\mathrm{b}} / \mathrm{d} t^{3}\left(\mathrm{~s}^{-2}\right) \quad 3.6(6) \times 10^{-28}$

Derivative of semi-major axis $\dot{x}\left(\mathrm{~s} \mathrm{~s}^{-1}\right)-23(3) \times 10^{-14}$

Upper limits to parameters ${ }^{b}$

$\begin{array}{ll}\text { Second derivative of } x, \ddot{x},\left(\mathrm{~s}^{-1}\right) & 7(15) \times 10^{-22} \\ \text { Derivative of eccentricity }|\dot{e}|\left(\mathrm{s}^{-1}\right) & 1 \times 10^{-12} \\ \text { Second Period deriv. } \ddot{P}\left(\mathrm{~s}^{-1}\right) & 5(2) \times 10^{-31} \\ \text { Second frequency derivative } \ddot{\nu}\left(\mathrm{s}^{-3}\right) & 2(1) \times 10^{-26}\end{array}$

\begin{tabular}{ll}
\multicolumn{2}{c}{ Deduced and additional parameters } \\
Distance $(D M-$ based $),(\mathrm{kpc})$ & 1.3 \\
Transverse velocity $V_{\mathrm{t}},\left(\mathrm{km} \mathrm{s}^{-1}\right)$ & $33(19)$ \\
Shklovskii effect $\dot{P}_{\mathrm{t}}\left(\mathrm{s} \mathrm{s}^{-1}\right)$ & $0.01 \div 0.09 \times 10^{-20}$ \\
Mass function $f_{\mathrm{m}}\left(M_{\odot}\right)$ & $0.99950(1) \times 10^{-5}$ \\
Companion mass $m_{\mathrm{c}}\left(M_{\odot}\right)$ & $0.0273 / \sin i^{c}$
\end{tabular}

rms TOA residual $\sigma(\mu \mathrm{s})$

Timing data span (MJD)

Number of TOAs

\section{1}

$49573-51908$

584

${ }^{a}$ See Fig. 4 for the values and uncertainties in proper motion.

$b$ These parameters were fixed at zero in global fit.

${ }^{c}$ Calculated assuming a pulsar mass $m_{\mathrm{p}}=1.4 \mathrm{M}_{\odot}$.

Note - Values in parenthesis are $1 \sigma$ uncertainties in the last quoted digit.

Laboratory (Standish 1990) and various relativistic timing models. Since the pulsar is moving in an almost circular orbit, we have applied the Blandford and Teukolsky phenomenological timing model (Blandford \& Teukolsky 1976). We have also fitted the model of Laplace-Lagrange parameters for systems with small eccentricity (Lange et al. 2001) using TEMPO. Comparing results obtained by TIMAPR and TEMPO are in excellent agreement within their uncertainties at a $1 \sigma$-level.

In the fitting procedure the three TOA segments, obtained with both the Effelsberg and Jodrell Bank data acquisition systems, were fitted for a mutual offset. Data lying in the range of orbital phases between 0.20 and 0.35

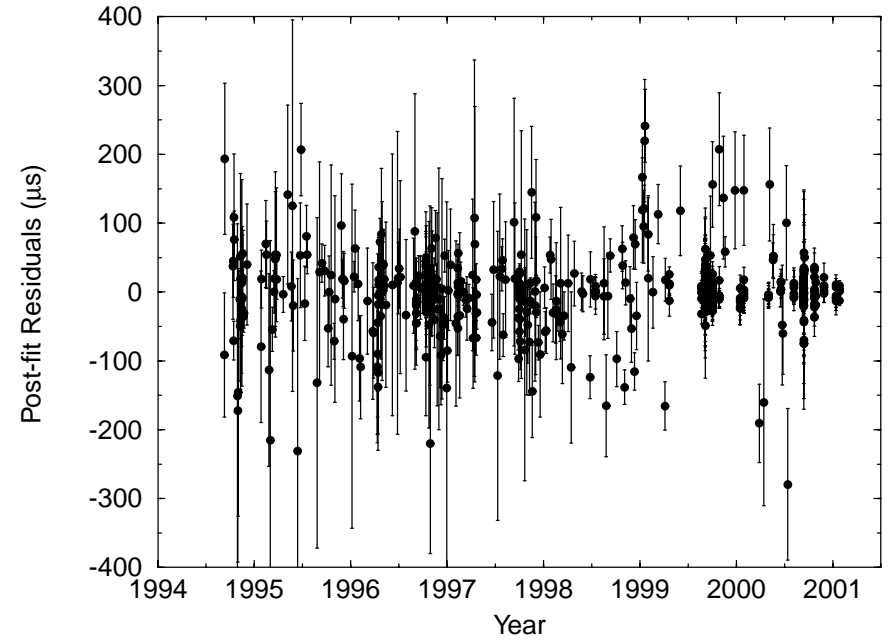

Fig. 2. Post-fit TOA residuals of PSR J2051-0827 plotted as a function of epoch, after subtracting a global 16-parameter timing model from the data.

were excluded from the fit because of the additional modulation of the excess column density on the TOAs in the eclipsing region (Stappers et al. 1998, 2001a). From the fit we obtained precise estimates of the pulsar's parameters and those of the orbit. These include astrometrical parameters, spin parameters, and Keplerian and post-Keplerian orbital parameters (Table 1).

Post-fit residuals are plotted as a function of observing epoch (Fig. 2) and as a function of orbital phase (Fig. 3). The upper limits on the values of $\dot{D} M, \ddot{x}, \dot{e}$ and $\ddot{P}$, presented in Table 1 , were obtained by the individual inclusion of the corresponding parameter in the fitting procedure. These parameters, as well as the longitude of periastron $\omega_{0}$, were fixed at zero while fitting for the other parameters.

Since the proper motion in right ascension and declination $\left(\mu_{\alpha}, \mu_{\delta}\right)$ has significant mutual covariances and the TOA uncertainties are relatively large, the global fit failed to give good estimates and uncertainties for $\mu_{\alpha}$ and $\mu_{\delta}$. To obtain more precise values of the proper motion parameters, we have mapped out the region of $\chi^{2}$ space near its global minimum in the $\left(\mu_{\alpha}, \mu_{\delta}\right)$ plane. Contours of $\Delta \chi^{2}\left(\mu_{\alpha}, \mu_{\delta}\right) \equiv \chi^{2}\left(\mu_{\alpha}, \mu_{\delta}\right)-\chi_{0}^{2}$, where $\chi_{0}^{2}$ is the minimum of the statistic $\chi^{2}$, are plotted in Fig. 4. They show regions of $1 \sigma\left(\Delta \chi^{2}=2.3\right), 2 \sigma\left(\Delta \chi^{2}=6.2\right)$ and $3 \sigma\left(\Delta \chi^{2}=11.8\right)$, which correspond to $68.3 \%, 95.4 \%$ and $99.7 \%$ confidence for $\mu_{\alpha}$ and $\mu_{\delta}$. Within $1 \sigma$ - uncertainty, the proper motion in right ascension is $\mu_{\alpha}=5.3 \pm 1.0$ mas yr$^{-1}$ and in dec-

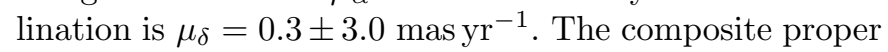
motion of PSR J2051-0827 is $|\mu|=5.3 \pm 3.0$ mas yr $^{-1}$.

The newly obtained celestial coordinates and binary parameters for PSR J2051-0827 are in excellent agreement to previously published values. The long time span even allows to detect higher order time derivatives of the orbital period $P_{\mathrm{b}}$ and the projected semi-major axis of the pulsar $x \equiv a_{\mathrm{p}} \sin i$. The measured values of $\dot{P}_{\mathrm{b}}, \ddot{P}_{\mathrm{b}}$ and $\dot{x}$ 


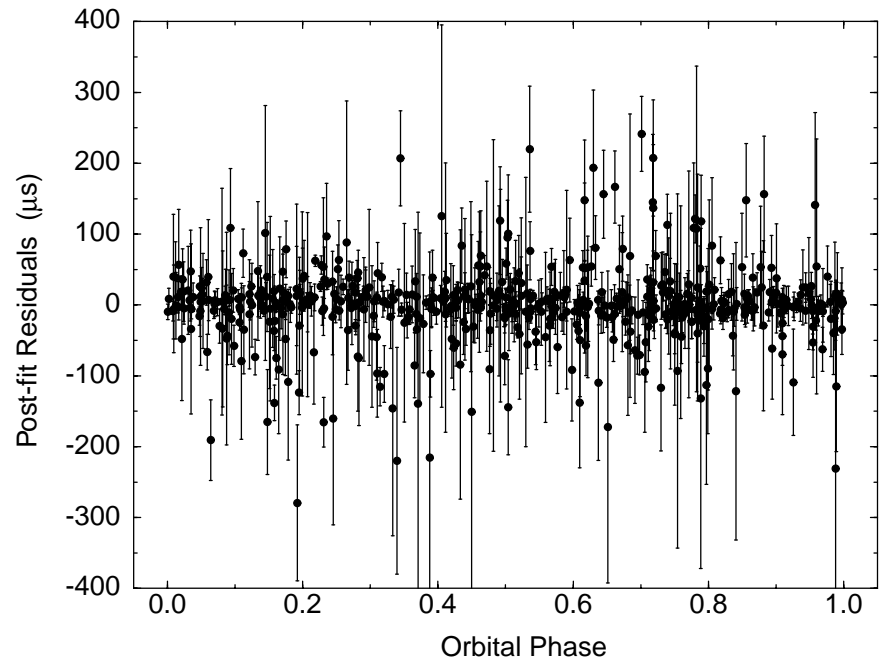

Fig. 3. Post-fit TOA residuals of PSR J2051-0827 plotted as a function of orbital phase of the pulsar in the binary system with $P_{\mathrm{b}} \simeq 2.4 \mathrm{hrs}$, after subtracting a global 16-parameter timing model from the data. The TOAs lying in the eclipsing region with the orbital phase $(0.20-0.35)$ were omitted from the global fit.

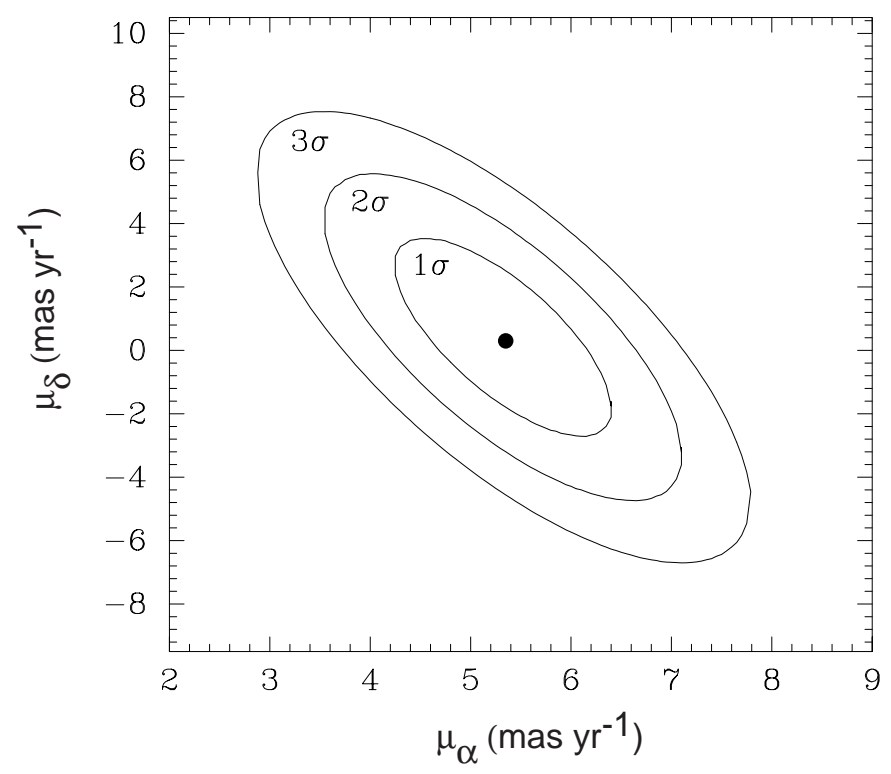

Fig. 4. Contours of $\chi^{2}$ near its global minimum in the $\left(\mu_{\alpha}, \mu_{\delta}\right)$ plane. The contours enclose regions of $68.3 \%, 95.4 \%$, and $99.7 \%$ confidence for values of $\mu_{\alpha}$ and $\mu_{\delta}$. The dot shows a low confidence value, corresponding to the minimal value of $\chi^{2}$.

are highly significant and were detected at a level of about $8 \sigma$, reducing the post-fit TOA residuals by $\sim 25 \%$.

\section{Variation of the pulsar orbit}

\subsection{Possible effects of $D M$ variations}

Variations of the dispersion measure, $D M$, which can be large in eclipsing binary systems, can have a significant influence on the TOAs and thus on the fitted orbital parameters and their time derivatives. Therefore we test if

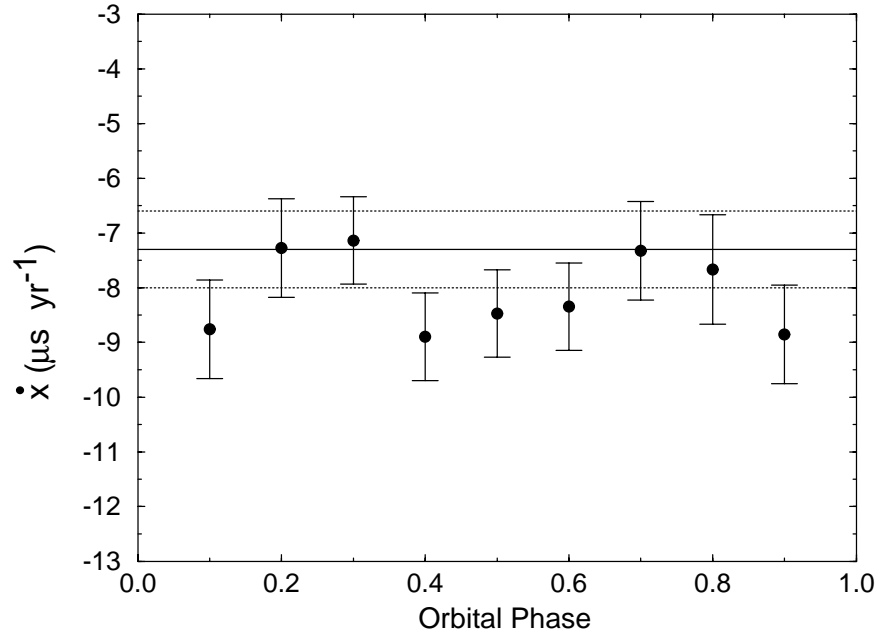

Fig. 5. Secular variations of the projected semi-major axis $x \equiv a_{\mathrm{p}} \sin i$ of PSR J2051-0827, obtained from fits of the data with excluded corresponding orbital phases. The solid line represents $\dot{x}=-7.3 \mu \mathrm{syr}^{-1}$ from Table 1 and the dotted lines show the uncertainty in $\dot{x}$.

the observed variations of the orbital parameters could be induced by $D M$ variations.

The best fit solution for PSR J2051-0827 reveals a secular variation of the dispersion measure at a rate $\mathrm{d}(D M) / \mathrm{d} t=0.0011(1) \mathrm{pc} \mathrm{cm}^{-3} \mathrm{yr}^{-1}$ (Table 1). This value is consistent with the expected square root dependence of $D M$ variations to the distance, caused by the motion of the pulsar through the interstellar medium (Backer et al. 1993). Apart from this long-term variation in $D M$, there might be variations over the orbit, which are due to a non-isotropic distribution of the electron column density $n_{\mathrm{e}}$ near the pulsar eclipsing region. The observed value of $\dot{x}$ may be caused by changes of $n_{\mathrm{e}}$ near regions of upper and lower conjunction. Such variations depend on the orbital phase. If the assumption about the variations in $n_{\mathrm{e}}$ near upper and lower conjunction is correct, the fit to the timing model using the TOAs with excluded data segments in the regions of upper and lower conjunction, should show no variation in the projected semi-major axis $x$. Figure 5 shows these sets of timing solutions for $\dot{x}$, where we have excluded $20 \%$ of the data around the corresponding orbital phases (e.g., in obtaining solution of $\dot{x}$ at orbital phase 0.3 we have excluded all TOAs, lying in range of phases $0.2-0.4)$. It is seen that the detected secular change in $x$ does not significantly depend onto the distribution of the electron column density along the orbit, so that $D M$ variations cannot explain the observed $\dot{x}$.

We have also performed a more general test of the nature of orbital parameter variations in a way described by Arzoumanian et al. (1994). The total data set was divided into five sub-intervals, each spanning about one year of TOAs. The individual TOA sub-sets were fitted for the pulsar spin and Keplerian parameters with variations of orbital parameters held fixed at zero. The time of periastron $T_{0}$ and the orbital period $P_{\mathrm{b}}$ were transformed to and held fixed at an epoch near the center of each 


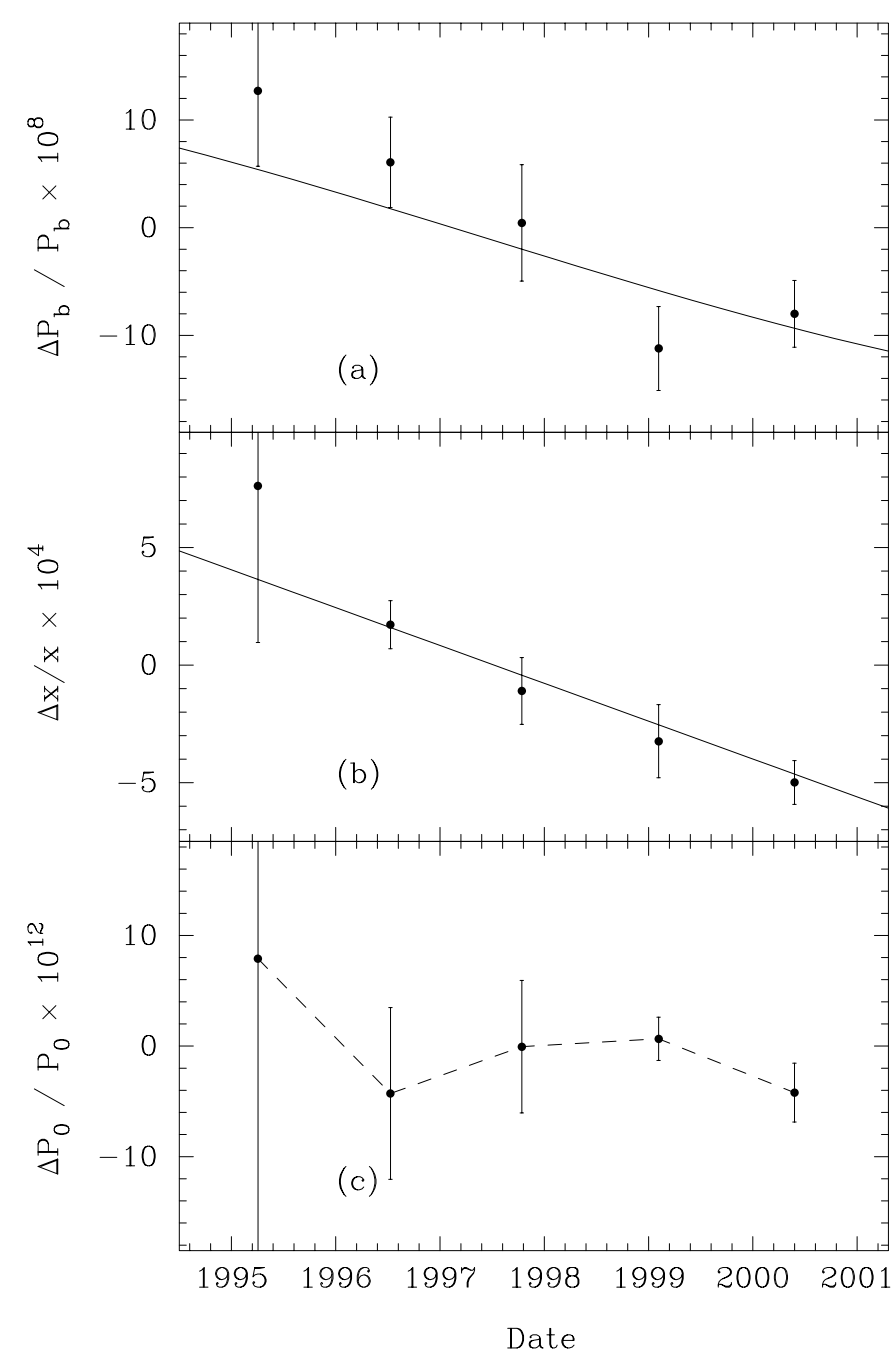

Fig. 6. a) Fractional changes of the orbital period in the PSR J2051-0827 system vs. date, obtained from fit of the pulsar parameters within four segments of data. b) Fractional changes of the projected semi-major axis of PSR J2051-0827 vs. date. c) Fractional pulse period changes vs. date. The solid lines corresponds to the values of $\dot{P}_{\mathrm{b}}, \ddot{P}_{\mathrm{b}}$ and $\dot{x}$ listed in Table 1 .

subset. The values of the pulsar spin period were transformed to the same epoch using the spin-down model of pulsar rotation, so that variations in period were calculated as $\Delta P_{0} \equiv \Delta P-\dot{P} \Delta t$. The resulting fractional changes in the orbital period, the projected semimajor axis, and the spin period are shown in Figs. 6a-c. Variations in $D M$ should affect measurements of the spin period $P_{0}$, orbital period $P_{\mathrm{b}}$ and semi-major axis $x$ in the same way as $\Delta P_{\mathrm{b}} / P_{\mathrm{b}}=\Delta x / x=\Delta P_{0} / P_{0}$. As relative changes differ by a few orders of magnitude, we conclude that the variations in $D M$ do not affect variations in $P_{\mathrm{b}}$, $x$ and $P$.

\subsection{Origin of the variation of the orbital period}

We have confirmed the existence of a large orbital period derivative of the PSR J2051-0827 binary system, previously reported by Stappers et al. (1998). The observed value is equal to $\dot{P}_{\mathrm{b}}=(-15.8 \pm 0.3) \times 10^{-12} \mathrm{~s} \mathrm{~s}^{-1}$ (see Table 1) and is in good agreement with that found by Stappers et al. (1998).

There are a number of effects that may cause the change of the orbital period of a binary system, which can be summarised as

$$
\left(\frac{\dot{P}_{\mathrm{b}}}{P_{\mathrm{b}}}\right)^{\mathrm{obs}}=\left(\frac{\dot{P}_{\mathrm{b}}}{P_{\mathrm{b}}}\right)^{\mathrm{GW}}+\left(\frac{\dot{P}_{\mathrm{b}}}{P_{\mathrm{b}}}\right)^{\mathrm{acc}}+\left(\frac{\dot{P}_{\mathrm{b}}}{P_{\mathrm{b}}}\right)^{\dot{m}}+\left(\frac{\dot{P}_{\mathrm{b}}}{P_{\mathrm{b}}}\right)^{\mathrm{T}}+\left(\frac{\dot{P}_{\mathrm{b}}}{P_{\mathrm{b}}}\right)^{Q}
$$

where the contributions to the observed orbital period derivative are due to the emission of gravitational radiation (GW), acceleration of the binary system (acc), mass loss from the system $(\dot{m})$, tidal dissipation of the orbit $(\mathrm{T})$, and gravitational quadrupole coupling $(Q)$.

The change in orbital period due to the general relativistic orbital decay,

$$
\begin{aligned}
\dot{P}_{\mathrm{b}}^{\mathrm{GW}}= & -(195 \pi / 5)\left(2 \pi T_{\odot} / P_{\mathrm{b}}\right)^{5 / 3}\left(1+\frac{73}{24} e^{2}+\frac{37}{96} e^{4}\right)(2) \\
& \times\left(1-e^{2}\right)^{-7 / 2}\left(m_{\mathrm{p}} m_{\mathrm{c}}\right) /\left(m_{\mathrm{p}}+m_{\mathrm{c}}\right)^{1 / 3}
\end{aligned}
$$

where $T_{\odot} \equiv G M_{\odot} / c^{3}=4.925490947 \times 10^{-6} \mathrm{~s}($ Damour \& Deruelle 1986; Damour \& Taylor 1992) and a pulsar mass of $m_{\mathrm{p}}=1.4 M_{\odot}$, is expected to be in the range $\dot{P}_{\mathrm{b}}^{\mathrm{GW}}=$ $(-6.9 \div-3.4) \times 10^{-14} \mathrm{~s} \mathrm{~s}^{-1}$ for the allowed companion masses $m_{\mathrm{c}}=(0.027 \div 0.055) M_{\odot}$. This value is about two orders of magnitude less than the observed value of $\dot{P}_{\mathrm{b}}$.

An acceleration of the binary system with respect to the Solar System Barycentre (SSB) may be caused by the differential rotation of the Galaxy or by a third massive body in the vicinity of the binary system. The acceleration affects the binary period derivative $\dot{P}_{\mathrm{b}}$ and the spin period derivative $\dot{P}$ in the same way. Even under the assumption that the observed value of the spin period derivative is totally due to acceleration, the contribution to the binary period derivative will be only $\left(\dot{P}_{\mathrm{b}} / P_{\mathrm{b}}\right)^{\text {acc }}=(\dot{P} / P)^{\text {acc }}=$ $3 \times 10^{-18} \mathrm{~s}^{-1}$, which is four orders of magnitude less than the observed value.

The mass loss from the binary system leads to a change in the orbital period at a rate (Damour \& Taylor 1991)

$\left(\frac{\dot{P}_{\mathrm{b}}}{P_{\mathrm{b}}}\right)^{\dot{m}}=\frac{8 \pi^{2}}{m_{\mathrm{p}}+m_{\mathrm{c}}} \frac{I_{\mathrm{a}}}{c^{2}} \frac{\dot{P}_{\mathrm{a}}}{P_{\mathrm{a}}^{3}}$,

where $I_{\mathrm{a}}$ denotes the moment of inertia of body a $(\mathrm{a}=\mathrm{p}$ for the pulsar, $\mathrm{a}=\mathrm{c}$ for the companion), $P_{\mathrm{a}}$ and $\dot{P}_{\mathrm{a}}$ are the spin period and period derivative. Since the companion is almost synchronously rotating around the pulsar $\left(P_{\mathrm{c}} \simeq P_{\mathrm{b}}\right)$, we can neglect its contribution. Using observed values of the pulsar spin period and its time derivative, and assuming $I_{\mathrm{p}} \sim 10^{45} \mathrm{~g} \mathrm{~cm}^{-2}$, the change of the orbital period due to mass loss is $\dot{P}_{\mathrm{b}}^{\dot{m}_{\mathrm{p}}} \sim 4 \times 10^{-17} \mathrm{~s} \mathrm{~s}^{-1}$ and hence too small to be of importance.

Tidal torques cannot be the reason for the observed $\dot{P}_{\mathrm{b}}$ either, because the magnitude of the tidal torque is orders of magnitudes too small to transfer the necessary angular momentum (Applegate 1992, and references therein). 
As all other contributions are several orders of magnitude smaller than the observed $\left(\dot{P}_{\mathrm{b}} / P_{\mathrm{b}}\right)$, we conclude that the change of the orbital period is most likely due to gravitational quadrupole coupling which has been proposed earlier for the eclipsing binary system PSR B1957+20 (Applegate \& Shaham 1994). A variable quadrupole moment which is due to a cyclic spin-up and spin-down of the outer layers of the companion, provides a natural explanation of the quasi-cyclic orbital variations of the PSR B1957+20 binary system found by Arzoumanian et al. (1994) and recently confirmed by Nice et al. (2000). In fact, our observations revealed that the orbital period derivative of PSR J2051-0827 changes with time at a rate of $\ddot{P}_{\mathrm{b}}=(+2.1 \pm 0.3) \times 10^{-20} \mathrm{~s}^{-1}$. This implies that the presently decreasing orbital period will increase after $\Delta t=-2 \dot{P}_{\mathrm{b}} / \ddot{P}_{\mathrm{b}} \sim 33$ yrs and that the system is undergoing quasi-cyclic variations like that found for PSR B1957+20. The orbital period change corresponding to a variation of the quadrupole moment of the companion, $\Delta Q$, is equal to (Applegate \& Shaham 1994)

$$
\left(\frac{\Delta P_{\mathrm{b}}}{P_{\mathrm{b}}}\right)^{Q}=-9 \frac{\Delta Q}{m_{\mathrm{c}} a^{2}},
$$

where $m_{\mathrm{c}}$ is the companion mass, and $a$ is the relative semi-major axis of the orbit $\left(a=a_{\mathrm{p}}+a_{\mathrm{c}}\right)$. The semimajor axes of the pulsar $a_{\mathrm{p}}=x / \sin i$ and companion $a_{\mathrm{c}}=x(1.4 / 0.0273) / \sin i$ are calculated for the pulsar and companion masses $m_{\mathrm{p}}=1.4 M_{\odot}$ and $m_{\mathrm{c}}=0.0273 M_{\odot}$ respectively. The quadrupole moment of the companion can be derived as (Kopal 1978)

$Q=\frac{2}{9} k \frac{\Omega_{\mathrm{c}}^{2} R_{\mathrm{c}}^{5}}{G}$,

where $k$ is the apsidal motion constant (Claret \& Gimenez $1991), \Omega_{\mathrm{c}}$ is the angular velocity of the companion, $R_{\mathrm{c}}$ its radius, and $G$ the Newtonian gravitation constant. By differentiating Eq. (5) the variation of the quadrupole moment with $\Omega_{\mathrm{c}}$ is

$$
\Delta Q=\frac{4}{9} k \frac{\Omega_{\mathrm{c}}^{2} R_{\mathrm{c}}^{5}}{G}\left(\frac{\Delta \Omega_{\mathrm{c}}}{\Omega_{\mathrm{c}}}\right) .
$$

From Eqs. (4) and (6) the variation of the companion angular velocity corresponding to the variation in the orbital period is derived as

$$
\left(\frac{\Delta \Omega_{\mathrm{c}}}{\Omega_{\mathrm{c}}}\right)=-\frac{1}{4}\left(\frac{\Delta P_{\mathrm{b}}}{P_{\mathrm{b}}}\right) m_{\mathrm{c}} a^{2} \frac{G}{k R_{\mathrm{c}}^{5} \Omega_{\mathrm{c}}^{2}} .
$$

We estimate that the radius of the pulsar's companion is $R_{\mathrm{c}} \sim 0.06 R_{\odot}$ (see Sect. 4.3). This value is equal to half the size of its Roche lobe $R_{\mathrm{L}}=0.13 R_{\odot}$ (Stappers et al. 1996a) and agrees with that inferred from optical observations of the companion (Stappers et al. 1999, 2000).

The apsidal motion constant, $k$, strongly depends on the effective temperature of the companion. The first optical observations indicated a temperature of $T_{\text {eff }}=4000 \div 4700 \mathrm{~K}$ (Stappers et al. 1996b) whereas recent observations with the HST indicated that the backside temperature is likely to be less then $3000 \mathrm{~K}$ (Stappers et al. 2000, 2001b). The corresponding value of the apsidal motion constant is $k=0.044 \div 0.159$ (Claret 2000 ). Since the companion spin frequency is close to that of the orbit, we can write for the general case

$\Omega_{\mathrm{c}}=f \frac{2 \pi}{P_{\mathrm{b}}}$

where $f$ is close to unity. While $f=1$ describes synchronous rotation, the wind and the magnetic activity of the companion in the Applegate \& Shaham (1994) model result in a torque which slows down companion's rotation, so that $f<1$. From the light curve observed by Stappers et al. (2001b, see their Fig. 1), we can expect this deviation from synchronous rotation to be small, estimating a lower limit of $f>0.9$. For $f \approx 1$, the change of the orbital period of $\Delta P_{\mathrm{b}} \sim-1.5 \times 10^{-4}$ s observed after six years is caused by a variation of the angular velocity of the companion of

$$
\left(\frac{\Delta \Omega_{\mathrm{c}}}{\Omega_{\mathrm{c}}}\right) \sim(8 \div 60) \times 10^{-4}
$$

for an orbital inclination $30^{\circ}<i<90^{\circ}$ and a range of companion masses of $m_{\mathrm{c}}=0.027 \div 0.055 M_{\odot}$. For $f \approx 0.9$ we derive $\Delta \Omega_{\mathrm{c}} / \Omega_{\mathrm{c}} \sim(1 \div 74) \times 10^{-3}$. These estimations of the angular velocity variations agree well with $\Delta \Omega_{\mathrm{c}} / \Omega_{\mathrm{c}} \sim 10^{-3}$ for the companion of PSR B1957+20 and with $\Delta \Omega_{\mathrm{c}} / \Omega_{\mathrm{c}} \sim(3 \div 30) \times 10^{-3}$ for Algol, RS CVn and CV systems (Applegate \& Shaham 1994).

\subsection{Origin of the variation of the projected semi-major axis}

We have detected a significant secular variation of the pulsar projected semi-major axis at a rate of $\dot{x} \equiv$ $\mathrm{d}(a \sin i) / \mathrm{d} t=(-23 \pm 3) \times 10^{-14} \mathrm{~s} \mathrm{~s}^{-1}$ in a global fit of the pulsar parameters. For an independent check of this effect, the TOA data set was split in five approximately equal segments, which were fitted for pulsar astrometric, spin and orbital parameters with the time derivatives of the orbital elements held fixed at zero. In these segments the epochs of ascending node were converted to values corresponding to the centre of each data set. The fitted values of the projected semi-major axis for these three time intervals are found to be in good agreement with the timing solution obtained from the global fit, and confirm the current decrease of the projected semi-major axis (Fig. 7).

The value of $\dot{x}$ cannot be explained by the observed time derivative in the orbital period $\dot{P}_{\mathrm{b}}$. The observed value of $\dot{P}_{\mathrm{b}}$ assumes a corresponding change in the projected semi-major axis as a consequence of Kepler's third law, at a rate

$\frac{\dot{x}}{x}=\frac{2}{3} \frac{\dot{P}_{\mathrm{b}}}{P_{\mathrm{b}}}$

For the measured values of $x, P_{\mathrm{b}}$ and $\dot{P}_{\mathrm{b}}$ one obtains $\dot{x}_{\max } \simeq-6 \times 10^{-17} \mathrm{~s} \mathrm{~s}^{-1}$, which is about 4 orders of magnitude less than the observed $\dot{x}$. 


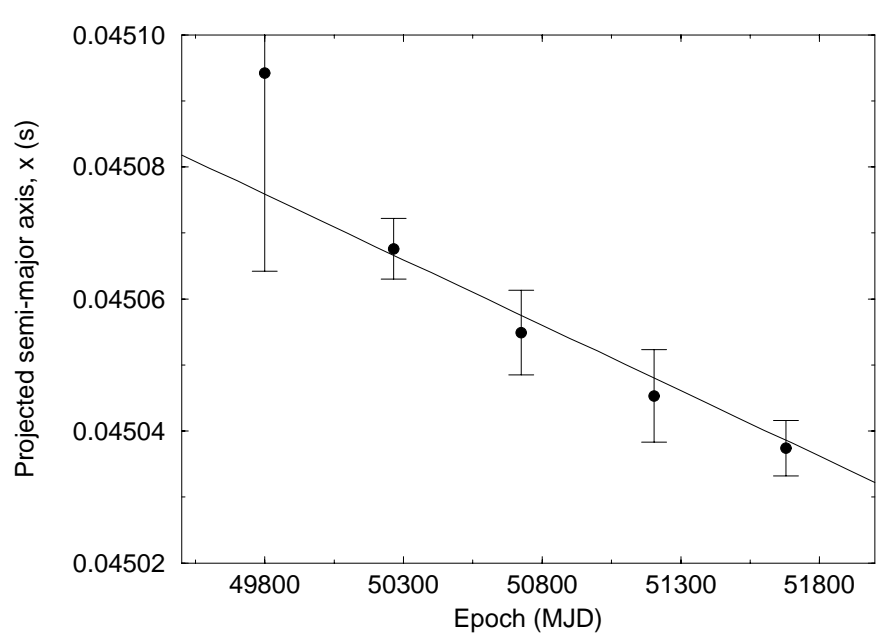

Fig. 7. Secular variation of the projected semi-major axis $x \equiv$ $a_{\mathrm{p}} \sin i$ of PSR J2051-0827. The dots indicate the values with their formal errors, obtained from fits of subsequent TOA data segments with the time derivatives of the orbital elements held fixed at zero. The solid line shows the secular variation of $x$ obtained from the global fit.

There are a few effects which may cause a secular change of the projected semi-major axis $x$ (Damour \& Taylor 1992; Kopeikin 1994; Wex \& Kopeikin 1999), i.e.

$$
\left(\frac{\dot{x}}{x}\right)^{\mathrm{obs}}=\left(\frac{\dot{a}_{\mathrm{p}}}{a_{\mathrm{p}}}\right)^{\mathrm{GW}}+\left(\frac{\dot{x}}{x}\right)^{\mathrm{PM}}+\frac{\mathrm{d} \varepsilon_{\mathrm{A}}}{\mathrm{d} t}-\frac{\dot{D}}{D}+\left(\frac{\dot{x}}{x}\right)^{\mathrm{SO}}
$$

where the contributions to the observed semi-major axis derivative are due to the emission of gravitational radiation $(\mathrm{GW})$, proper motion of the binary system (PM), varying aberration $\left(\mathrm{d} \varepsilon_{\mathrm{A}} / \mathrm{d} t\right)$, changing Doppler shift $(-\dot{D} / D)$ and spin-orbit coupling (SO) in the binary system.

The first contribution (GW) in Eq. (11) represents the shrinking of the pulsar orbit due to gravitational-wave damping in the binary system given by

$$
\left(\frac{\dot{a}_{\mathrm{p}}}{a_{\mathrm{p}}}\right)^{\mathrm{GW}}=\frac{2}{3}\left(\frac{\dot{P}_{\mathrm{b}}}{P_{\mathrm{b}}}\right)^{\mathrm{GW}} \text {. }
$$

With $\dot{P}_{\mathrm{b}}^{\mathrm{GW}}$ calculated from Eq. (2), one expects a rate of $\left(\dot{a}_{\mathrm{p}} / a_{\mathrm{p}}\right)^{\mathrm{GW}} \sim(-5.4 \div-2.6) \times 10^{-18} \mathrm{~s}^{-1}$, which is about 6 orders of magnitude smaller than the observed $(\dot{x} / x)$ and can be neglected.

The second contribution (PM) in Eq. (11) is caused by the proper motion of the binary system (Kopeikin 1996; Arzoumanian et al. 1996; Bell et al. 1997) given by

$$
\left(\frac{\dot{x}}{x}\right)^{\mathrm{PM}}=1.54 \times 10^{-16} \cot i\left(-\mu_{\alpha} \sin \Omega+\mu_{\delta} \cos \Omega\right),
$$

where $\Omega$ is the longitude of the ascending node of the orbit, and $\mu_{\alpha}$ and $\mu_{\delta}\left(\right.$ mas $\left.\mathrm{yr}^{-1}\right)$ are the proper motions in right ascension and declination. In this case, the observed change in $x$ is caused by the secular variation in the inclination angle $i$ due to the proper motion, whereas the intrinsic semi-major axis of the pulsar $a_{\mathrm{p}}$ remains constant. The maximal contribution of the proper motion is

$$
\left(\frac{\dot{x}}{x}\right)_{\max }^{\mathrm{PM}} \simeq 1.54 \times 10^{-16} \mu \cot i
$$

with a composite proper motion $\mu=\left(\mu_{\alpha}^{2}+\mu_{\delta}^{2}\right)^{1 / 2}=$ $5(3)$ mas $_{\mathrm{yr}^{-1}}$. In order to estimate this quantity we still need an estimation of the maximal inclination angle $i$.

If the companion is a white dwarf, we obtain a minimal radius using the mass-radius relation of Paczyński (1967) of $R_{\mathrm{c} \text { min }}=0.03 R_{\odot}$ for mass ranges of $m_{\mathrm{c}}=$ $0.027 \div 0.055 M_{\odot}$. If the companion is, instead, a nondegenerate star in the pulsar radiation field, as suggested by Ergma et al. (1998), the radius is even larger. We hence obtain $R_{\text {c min }}=0.03 R_{\odot}$ as a safe lower limit. Since at higher frequencies the pulsar signal is not eclipsed by the companion that is separated by $1.03 R_{\odot}$, we derive an upper limit for the inclination angle of $i_{\max }=88^{\circ}$. This inclination angle leads to $\dot{x}_{\max }^{\mathrm{PM}}=1.2(7) \times 10^{-18} \mathrm{~s} \mathrm{~s}^{-1}$. For the likely inclination of $i \sim 40^{\circ}$ (Stappers et al. 1999) we obtain $\dot{x}^{\mathrm{PM}}=4(2) \times 10^{-17} \mathrm{~s} \mathrm{~s}^{-1}$. This is at least four orders of magnitude smaller than the observed $\dot{x}$ and can be neglected.

The third term, $\left(\mathrm{d} \varepsilon_{\mathrm{A}} / \mathrm{d} t\right)$, in Eq. (11) is due to varying aberration caused by relativistic precession of the pulsar spin axis

$$
\frac{\mathrm{d} \varepsilon_{\mathrm{A}}}{\mathrm{d} t}=-\frac{P}{P_{\mathrm{b}}} \frac{\Omega_{\mathrm{p}}^{\mathrm{geod}}}{\left(1-e^{2}\right)^{1 / 2}} \frac{(\cot \lambda \sin 2 \eta+\cot i \cos \eta)}{\sin \lambda},
$$

where $P$ is the pulsar spin period, and $(\eta, \lambda)$ are the polar coordinates of the pulsar spin (see Damour \& Taylor 1992). The rate of geodetic precession given by (Barker \& O'Connel 1975)

$\Omega_{\mathrm{p}}^{\mathrm{geod}}=\frac{G^{2 / 3}}{2 c^{2}\left(1-e^{2}\right)}\left(\frac{2 \pi}{P_{\mathrm{b}}}\right)^{5 / 3} \frac{m_{\mathrm{c}}\left(4 m_{\mathrm{p}}+3 m_{\mathrm{c}}\right)}{\left(m_{\mathrm{p}}+m_{\mathrm{c}}\right)^{4 / 3}}$

amounts to $\Omega_{\mathrm{p}}^{\mathrm{geod}} \sim 1.7 \times 10^{-10}=0.3^{\circ} \mathrm{yr}^{-1}$ for a companion mass of $m_{\mathrm{c}}=0.055 M_{\odot}$. This leads to $\left(\mathrm{d} \varepsilon_{A} / \mathrm{d} t\right)<$ $8.5 \times 10^{-20} \mathrm{~s}^{-1}$, which is about 6 orders of magnitude less than the observed $(\dot{x} / x)$ and can be neglected.

The fourth term in Eq. (11), $(\dot{D} / D)$, is due to a changing Doppler shift $(D)$ caused by the change of the distance between the SSB and the binary pulsar system due to the acceleration of the binary system in the gravitational field of the Galaxy and due to the proper motion on the plane of the sky (Shklovskii 1970; Damour \& Deruelle 1986). We obtain

$-\frac{\dot{D}}{D}=\frac{1}{c} \boldsymbol{K}_{0} \cdot\left(\boldsymbol{a}_{\mathrm{PSR}}-\boldsymbol{a}_{\mathrm{SSB}}\right)+\frac{V_{\mathrm{T}}^{2}}{c d}$,

where $\boldsymbol{K}_{0}$ is the unit vector of the pulsar along the line of sight, $\boldsymbol{a}_{\mathrm{PSR}}$ and $\boldsymbol{a}_{\mathrm{SSB}}$ are the Galactic accelerations at the location of the PSR J2051-0827 binary system and the $\mathrm{SSB}, V_{\mathrm{T}}$ the transverse velocity of the pulsar, and $d$ the distance between the pulsar and the SSB. Using an estimation of $\boldsymbol{a}_{\mathrm{SSB}} \sim 2.1 \times 10^{-7} \mathrm{~m} \mathrm{~s}^{-2}$ 
(Carlberg \& Innanen 1987) we derive for the first term in Eq. (17) an upper limit of $7 \times 10^{-16} \mathrm{~s}^{-1}$. The transverse velocity of the pulsar, $V_{\mathrm{T}}=33 \mathrm{~km} \mathrm{~s}^{-1}$, and a distance to the pulsar of $d=1.3 \mathrm{kpc}$ yield a value of $V_{\mathrm{T}}^{2} / c d \sim 10^{-19} \mathrm{~s}^{-1}$. Thus, the total contribution from a varying Doppler shift to the observed $(\dot{x} / x)$ is less than $0.3 \%$ and can be neglected.

As all other contributions are several orders of magnitude smaller than the observed $(\dot{x} / x)$, the variation of $x$ is most likely caused by classical spin-orbit coupling ( $\mathrm{SO}$ ) in the binary system (Eq. (11)). Spin-orbit coupling leads to a variation in the inclination angle, $(\mathrm{d} i / \mathrm{d} t)$, while the semi-major axis $a_{\mathrm{p}}$ remains constant. In this case the observed value $(\mathrm{d} i / \mathrm{d} t)$ is

$\frac{\mathrm{d} i}{\mathrm{~d} t}=\left(\frac{\dot{x}}{x}\right)^{\mathrm{SO}} \tan i$.

If we assume that the observed $\dot{x}$ is totally due to spinorbit coupling, we obtain $(\mathrm{d} i / \mathrm{d} t)=-0.008^{\circ} \mathrm{yr}^{-1}$ for an orbital inclination of $i=40^{\circ}$ and $(\mathrm{d} i / \mathrm{d} t)=-0.26^{\circ} \mathrm{yr}^{-1}$ for $i=88^{\circ}$.

The rotationally induced quadrupole of the companion produces a variation of the orbital inclination angle as (Lai et al. 1995; Wex 1998)

$\frac{\mathrm{d} i}{\mathrm{~d} t}=\frac{1}{2} \Omega_{\mathrm{orb}} \frac{k R_{\mathrm{c}}^{2} \hat{\Omega}_{s}^{2}}{a^{2}\left(1-e^{2}\right)^{2}} \sin (2 \theta) \sin \Phi$.

Here $\Omega_{\mathrm{orb}} \equiv 2 \pi / P_{\mathrm{b}}$ is the orbital frequency and $\hat{\Omega}_{\mathrm{s}} \equiv$ $\Omega_{\mathrm{c}} /\left(G m_{\mathrm{c}} / R_{\mathrm{c}}^{3}\right)^{1 / 2}$ the dimensionless spin of the companion, $R_{\mathrm{c}}$ its radius, $a$ the semi-major axis of the relative orbit, $k$ the apsidal motion constant, $\theta$ the angle between the companion spin angular momentum $\boldsymbol{S}$ and the orbital angular momentum $\boldsymbol{L}$, and $\Phi$ the orbital plane precessional phase. Equation (19) is valid for the case that $|\boldsymbol{S}| \ll|\boldsymbol{L}|$, which is true for PSR J2051-0827, where $|\boldsymbol{S}| \sim 10^{-3}|\boldsymbol{L}|$. Note that spin-orbit coupling requires the companions's spin axis to be inclined with respect to the orbital angular momentum vector $(\theta \neq 0)$. Measurement of $(\mathrm{d} i / \mathrm{d} t)$ and the classical periastron advance $\dot{\omega}$ due to spin-orbit coupling can in principle be used to obtain constraints of the values of $\theta$ and $\Phi$ (Kaspi et al. 1996; Wex 1998). Unfortunately, the PSR J2051-0827 system has a negligible small eccentricity and all effects of secular variations in $\omega$, along with the substantial relativistic advance of periastron $\dot{\omega}^{\mathrm{GR}} \sim 12^{\circ} \mathrm{yr}^{-1}$, are fully absorbed by the redefinition of the binary period. Therefore, they are not observable in this system (Kopeikin \& Ozernoy 1999), so that we cannot obtain constraints from measurements of $\dot{\omega}$. Nevertheless we can yield an upper limit for $(\mathrm{d} i / \mathrm{d} t)$ and thus for the companion's radius from Eq. (18), i.e.

$\left(\frac{\mathrm{d} i}{\mathrm{~d} t}\right)_{\max }=\left(\frac{\dot{x}_{\max }}{x_{\min }}\right) \tan i_{\max }$,

where the indices max, min refer to the maximal or minimal value of the corresponding parameter substituting observed values of $\dot{x}_{\max }$ and $x_{\min }$ from Table 1 . Using the maximal inclination $i_{\max }=88^{\circ}$, the corresponding upper $\operatorname{limit}(\mathrm{d} i / \mathrm{d} t)_{\max } \sim-1.7 \times 10^{-10} \mathrm{~s}^{-1}, k=0.044 \div 0.159$, $a=1.03 R_{\odot}$ and $e=0$, we then obtain for the case of a synchronously rotating companion $\left(\Omega_{\mathrm{c}}=\Omega_{\text {orb }}\right)$ a maximal companion radius of $R_{\mathrm{c} \max } \sim 0.05 R_{\odot}$. According to optical observations, the system is likely to be moderately inclined with an inclination angle $i \sim 40^{\circ}$ (Stappers et al. 2000, 2001b) which would lead to $(\mathrm{d} i / \mathrm{d} t)_{\max } \sim$ $-4.8 \times 10^{-12} \mathrm{~s}^{-1}$ and a maximal companion radius of $R_{\text {c } \max } \sim 0.03 R_{\odot}$. The latter value is even smaller than the minimal radius for a pure helium white dwarf companion (Paczyński 1967) and therefore not very likely. If the companion is not perfectly synchronously rotating $\left(f \approx 0.9\right.$, see Eq. $(8)$ ), we obtain $R_{\mathrm{c} \max } \sim 0.04 R_{\odot}$ for $i \sim 40^{\circ}$ and $R_{\mathrm{c} \max } \sim 0.06 R_{\odot}$ for $i \sim 88^{\circ}$. A radius of $R_{\mathrm{c}} \sim 0.06 R_{\odot}$ is equal to half the size of its Roche lobe $R_{\mathrm{L}}=0.13 R_{\odot}$ (Stappers et al. 1996a) and agrees well with that inferred from optical observations of the companion (Stappers et al. 1999, 2000).

\section{Discussion and conclusions}

The detected quasi-cyclic orbital variations of the PSR J2051-0827 binary system are most likely caused by a variable quadrupole moment of the companion. For PSR B1957+20 Applegate \& Shaham (1994) suggested that the companion's wind due to the pulsar irradiation and its magnetic activity result in a torque that forces the companion slightly out of synchronous rotation. We propose that this mechanism is the likely source of orbital variability also in the PSR J2051-0827 binary system. In this framework the resulting tidal dissipation of energy is the source of the magnetic activity that causes the cyclic spin-up and spin-down of the outer layers of the companion. This makes the companion of PSR J2051-0827 the only second identified tidally powered star. We conclude that the companion is at least partially non-degenerate, convective and magnetically active (cf. Arzoumanian et al. 1994) in accordance with a model proposed by D'Antona \& Ergma (1993).

The torque acting on the companion in the Applegate \& Shaham (1994) model causes small deviations from a perfect co-rotation $(f<1)$. This situation may be an explanation for the slight asymmetry in the companion's light curve that was recently observed by Stappers et al. (2001b). We note, however, that even though a similar asymmetry detected for the companion of PSR B1957+20 by Djorgovski \& Evans (1988) could not be confirmed by later observations (Callanan et al. 1989), it was first interpreted as the result of a shock caused by the interaction of the pulsar and companion winds. A similar situation may be present in PSR J2051-0827, but the non-perfect corotation could well play an important role in deciphering the observed light curve.

The variation of the projected semi-major axis of the pulsar can be explaind by classical spin-orbit coupling. Due to a significant quadrupole moment of the companion the spin and orbital angular momenta, which are not aligned, couple and precess about the fixed total 
angular momentum vector. The precession of the orbit results in the observed variation of the inclination angle whereas the periastron advance cannot be detected due to the extremely small eccentricity of the system. The misalignment of the present system provides evidence that the neutron star received a kick at birth (cf. Kaspi et al. 1996) presenting further proof for the existence of asymmetric supernova explosions. A more detailed study will be presented elsewhere (Löhmer et al., in prep.).

Using timing information we are able to obtain a maximal radius of the companion of $R_{\mathrm{c} \max } \sim 0.06 R_{\odot}$, which is about half the size of its Roche lobe $R_{\mathrm{L}}=0.13 R_{\odot}$. This value is consistent with that obtained by modelling the light curve of the companion (Stappers et al. 2001b).

The quasi-cyclic variations of the orbital period show that there is no secular orbital decay leading to a destruction of the binary system, which has been discussed, based on previous timing data, by Stappers et al. (1998). As there is no Roche lobe overflow and the timescale to evaporate the companion by the pulsar's relativistic wind is $\sim 10^{9}$ yr (Stappers et al. 1998) the PSR J2051-0827 binary system is not likely to become an isolated millisecond pulsar in the future.

Acknowledgements. We are very grateful to all people involved in the pulsar timing project in Effelsberg, in particular to Karl Grypstra for maintaining the observatory clocks, and to the telescope operators at Effelsberg. We thank Norbert Wex and Aris Karastergiou for their assistance in observations. O.D. gratefully acknowledges the Alexander von Humboldt-Stiftung for supporting this work and acknowledges the receipt of the Max-Planck-Gesellschaft contract.

\section{References}

Applegate, J. H. 1992, ApJ, 385, 621

Applegate, J. H., \& Shaham, J. 1994, ApJ, 436, 312

Arzoumanian, Z., Fruchter, A. S., \& Taylor, J. H. 1994, ApJ, $426, \mathrm{~L} 85$

Arzoumanian, Z., Joshi, K., Rasio, F. A., \& Thorsett, S. E. 1996, in Pulsars: Progress and Problems, ed. S. Johnston, M. A. Walker, \& M. Bailes (San Francisco: ASP), ASP Conf. Ser. 105, IAU Colloq., 160, 525

Backer, D. C., Hama, S., van Hook, S., \& Foster, R. S. 1993, ApJ, 404, 636

Backer, D. C, Dexter, M. R., Zepka, A., et al. 1997, PASP, 109, 61

Barker, B. M., \& O'Connel, R. F. 1975, ApJL, 199, L25

Bell, J. F., Bailes, M., Manchester, R. N., et al. 1997, MNRAS, 286, 463

Bhattacharya, D., \& van den Heuvel, E. P. J. 1991, Phys. Rep., 203, 1
Blandford, R. D., \& Teukolsky, S. A. 1976, ApJ, 205, 580

Carlberg, R. C., \& Innanen, K. A. 1987, AJ, 94, 666

Callanan, P. J., Charles, P. A., \& van Paradijs, J. 1989, MNRAS, 240, 31

Claret, A., \& Gimenez, A. 1991, A\&AS, 87, 507

Claret, A. 2000, A\&A, 359, 289

D'Antona, F., \& Ergma, E. 1993, A\&A, 269, 219

Damour, T., \& Deruelle, N. 1986, Ann. Inst. Henri Poincaré A (Physique Théorique), 44, 263

Damour, T., \& Taylor, J. H. 1991, ApJ, 366, 501

Damour, T., \& Taylor, J. H. 1992, Phys. Rev. D., 45, 1840

Djorgovski, S., \& Charles, R. E. 1988, ApJ, 335, L61

Doroshenko, O. V., \& Kopeikin, S. M. 1995, MNRAS, 271, 1029

Ergma, E., Sarna, M. J., \& Antipova, J. 1998, MNRAS, 300, 352

Kaspi, V. M., Bailes, M., Manchester, R. N., Stappers, B. W., \& Bell, J. F. 1996, Nature, 381, 584

Kopal, Z. 1978, Dynamics of Close Binary Systems (D. Reidel Publishing Company, Dordrecht)

Kopeikin, S. M. 1994, ApJ, 434, L67

Kopeikin, S. M. 1996, ApJ, 467, L93

Kopeikin, S. M., \& Ozernoy, L. M. 1999, ApJ, 523, 771

Kramer, M. 1994, A\&AS, 107, 527

Kramer, M., Xilouris, K. M., Jessner, A., et al. 1997, A\&A, 322,846

Kramer, M., Xilouris, K. M., Lorimer, D. R., et al. 1998, ApJ, 501, 270

Lai, D., Bildsten, L., \& Kaspi, V. M. 1995, ApJ, 452, 819

Lange, C., Camilo, F., Wex, N., et al. 2001, MNRAS, 326, 274

Nice, D. J., Arzoumanian, Z., \& Thorsett, S. E. 2000, in Pulsar Astronomy - 2000 and Beyond, ed. M. Kramer, N. Wex, \& N. Wielebinski, ASP Conf. Ser. 202, IAU Colloq. 177 (San Francisco: ASP), 67

Paczyński, B. 1967, Acta Astr., 17, 287

Shklovskii, I. S. 1970, SvA, 13, 582

Standish, E. M. Jr. 1990, A\&A, 233, 252

Stappers, B. W., Bailes, M., Lyne, A. G., et al. 1996a, ApJ, 465, L119

Stappers, B. W., Bessel, M. S., \& Bailes, M. 1996b, ApJ, 473, L119

Stappers, B. W. Bailes, M., Manchester, R. N., Sandhu, J. S., \& Toscano, M. 1998, ApJ, 499, L183

Stappers, B. W., van Kerkwijk, M. H., Lane, B., \& Kulkarni, S. 1999, ApJ, 510, L45

Stappers, B. W., van Kerkwijk, M., \& Bell, J. F. 2000, in Pulsar Astronomy - 2000 and Beyond, ed. M. Kramer, N. Wex, \& N. Wielebinski, ASP Conf. Ser. 202, IAU Colloq. 177 (San Francisco: ASP), 627

Stappers, B. W., Bailes, M., Lyne, A. G., et al. 2001a, MNRAS, 321,576

Stappers, B. W., van Kerkwijk, M., Bell, J. F., \& Kulkarni, S. R. 2001b, ApJ, 548, L183

Taylor, J. H. 1991, Proc. IEEE, 79, 1054

Wex, N., \& Kopeikin, S. M. 1999, ApJ, 514, 388

Wex, N. 1998, MNRAS, 298, 67 